\title{
BMJ Open Rates of turnover among general practitioners: a retrospective study of all English general practices between 2007 and 2019
}

\author{
Rosa Parisi, ${ }^{1,2}$ Yiu-Shing Lau, ${ }^{3}$ Peter Bower, ${ }^{2}$ Kath Checkland, ${ }^{2,3}$ Jill Rubery, ${ }^{4}$ \\ Matt Sutton, ${ }^{3}$ Sally J Giles, ${ }^{5}$ Aneez Esmail, ${ }^{2}$ Sharon Spooner (i) ,,3 \\ Evangelos Kontopantelis (D) 1,2
}

To cite: Parisi R, Lau Y-S, Bower $\mathrm{P}$, et al. Rates of turnover among general practitioners: a retrospective study of all English general practices between 2007 and 2019. BMJ Open 2021;11:e049827. doi:10.1136/ bmjopen-2021-049827

- Prepublication history and additional supplemental material for this paper are available online. To view these files, please visit the journal online (http://dx.doi.org/10.1136/ bmjopen-2021-049827)

Received 04 February 2021 Accepted 12 July 2021
Check for updates

(C) Author(s) (or their employer(s)) 2021. Re-use permitted under CC BY-NC. No commercial re-use. See rights and permissions. Published by BMJ.

For numbered affiliations see end of article.

Correspondence to

Professor Evangelos

Kontopantelis;

e.kontopantelis@manchester. ac.uk

\section{ABSTRACT}

Objective To quantify general practitioners' (GPS') turnover in England between 2007 and 2019, describe trends over time, regional differences and associations with social deprivation or other practice characteristics. Design A retrospective study of annual cross-sectional data.

Setting All general practices in England (8085 in 2007, 6598 in 2019).

Methods We calculated turnover rates, defined as the proportion of GPs leaving a practice. Rates and their median, 25th and 75th percentiles were calculated by year and region. The proportion of practices with persistent high turnover (>10\%) over consecutive years were also calculated. A negative binomial regression model assessed the association between turnover and social deprivation or other practice characteristics.

Results Turnover rates increased over time. The 75th percentile in 2009 was $11 \%$, but increased to $14 \%$ in 2019. The highest turnover rate was observed in 20132014 , corresponding to the 75 th percentile of $18.2 \%$. Over time, regions experienced increases in turnover rates, although it varied across English regions. The proportion of practices with high (10\% to $40 \%$ ) turnover within a year almost doubled from 14\% in 2009 to $27 \%$ in 2019 . A rise in the number of practices with persistent high turnover $(>10 \%)$ for at least three consecutive years was also observed, from $2.7 \%(2.3 \%-3.1 \%)$ in 2007 to $6.3 \%$ $(5.7 \%-6.9 \%)$ in 2017. The statistical analyses revealed that practice-area deprivation was moderately associated with turnover rate, with practices in the most deprived area having higher turnover rates compared with practices in the least deprived areas (incidence rate ratios 1.09; $95 \% \mathrm{Cl} 1.06$ to 1.13 ).

Conclusions GP turnover has increased in the last decade nationally, with regional variability. Greater attention to GP turnover is needed, in the most deprived areas in particular, where GPs often need to deal with more complex health needs. There is a large cost associated with GP turnover and practices with very high persistent turnover need to be further researched, and the causes behind this identified, to allow support strategies and policies to be developed.

\section{Strengths and limitations of this study}

- This study goes a step further than previous research, in quantifying and describing general practitioners' (GPs') actual turnover over a 12-year period, rather than 'intention to leave'.

- It used two national administrative datasets, regularly updated and monitored, which have the advantage of including everyone rather than only respondents to a survey.

- It also presented methodological advances in combining multiple data sources containing information about the primary care workforce and historical data of individual GPs' characteristics.

- However, only a limited set of covariates was available in the national administrative datasets, when many more are relevant to turnover.

- Finally, it was not possible to distinguish between those GPs who moved to a different practice, retired or left primary care completely.

\section{INTRODUCTION}

Primary care has a key role in the UK healthcare system, with general practitioners (GPs, family physicians in the USA) the first point of contact for patient care. However, recent data have shown that the GP workforce in England is going through a major crisis, ${ }^{1}$ reflected in increasing rates of early retirement and intentions to reduce hours of working or leave their practice in the near future. ${ }^{2}$ Despite this being a common problem for other European countries ${ }^{3}$ and globally, ${ }^{4}$ it seems to be particularly serious for the UK. ${ }^{35}$ According to an international survey of GPs from 2015, approximately $30 \%$ of GPs want to leave their profession within 5 years. ${ }^{3}$

A 2019 report conducted by the Health Foundation highlighted that while there has been an increase in the number of hospitalbased doctors, the number of GPs has reduced $^{6}$; National Health Service (NHS) 
staff retention has worsened since 2011/2012 and, despite this being a UK government priority, there has been no improvement in retention in recent years. ${ }^{6}$ Prior to the 2015 elections, the UK government promised 5000 more doctors in primary care by $2020{ }^{7}$ However, recent data from regional and national surveys indicate the number of full-time equivalent GPs per 1000 patients continues to decline. Regionally, GP surveys from West Midlands ${ }^{8}$ and South West England ${ }^{9}$ found that $41.9 \%$ and $70 \%$ of participants intended to leave the practice or were likely or very likely to pursue a career choice that would negatively impact the GP workforce within the next 5 years, respectively. Likewise, the most recent national survey of 2195 GPs in England conducted in 2017 reported that $39 \%$ intended to leave 'direct patient care' within 5 years, compared with $19.4 \%$ in $2005 .^{2}$

GP retention measures the percentage of staff staying in a practice for a defined period of time. GP turnover measures the proportion of staff who leave. Both are important indicators of the behaviour of doctors in the primary care workforce. ${ }^{6}$ While retention is an indicator of the stability of a practice workforce, GP turnover is highly correlated with the desire to quit the profession, although this may in part be due to GPs retiring or simply moving practice.

Low retention or decreasing retention levels over time and high turnover rates are major issues for NHS primary care. High GP turnover is a concern for several reasons: it may be associated with practices experiencing recurring problems with recruitment and retention ${ }^{10}$; it may affect the ability to deliver primary care services ${ }^{4}$ and undermine continuity of care which in turn may affect the quality of patient care. For instance, healthcare received from multiple GPs can lead to conflicting therapeutic treatments and fragmented care. ${ }^{11}$ Conversely, the benefits of continuity of care have been documented in studies which linked greater continuity of care with higher patient satisfaction, ${ }^{12}$ reduction in costs of care, ${ }^{13}$ reduced risk of hospitalisation and lower mortality. ${ }^{14}$ Differential turnover across practices and regions could also lead to a maldistribution of GPs, exacerbating retention problems ${ }^{10}$ and health inequalities. It is also important to highlight that there is a large cost associated with GP turnover, ${ }^{15}$ estimated to be two to three times the doctor's annual salary. ${ }^{16}$ These costs include direct costs (separation costs, recruitment, induction and temporary replacement costs), ${ }^{17}$ but also indirect or long-term costs such as overwork by other staff plus the 'costs' in terms of quality of care. For instance, lower quality of care may lead to fewer patients seeking early diagnosis/treatments with long-term costs for the NHS as a whole. Finally, GP turnover costs are likely to increase in the future due to the GP shortages which are linked but not necessarily are a consequence of turnover.

Despite existing concerns about retention and turnover levels in England, studies quantifying movements of the GP workforce are scarce with the most recent reporting data from the early $90 \mathrm{~s} .{ }^{10}$ Recently, Buchan et al in their report conclude that further research is required particularly to investigate actual turnover as opposed to intentions to leave. ${ }^{618}$

In England, detailed administrative data about the primary care workforce are collected and include practicerelated characteristics as well as historical data on when a GP joins and leaves a practice. Compared with surveys, administrative data have the advantage that everyone is included rather than only the respondents and it is based on actual behaviour rather than intentions. However, these data have rarely been used to quantify actual GP turnover rates.

In this study, we used national data from NHS Digital, NHS Prescribing and the NHS Organisation Data Service (ODS) to explore GP turnover rates over time and regionally, as well as to identify practice-level factors associated with them.

\section{METHODS}

The overall aim of the study was to explore turnover rates of GPs and look at trends in turnover in different regions over time in England between 2007 and 2019. In particular, the study aimed to: quantify rates of GP turnover in England, their trends over time, their differences across regions and the predictors of GP turnover.

\section{Definition of GP turnover rates}

With the aim of quantifying trends of GPs leaving general practices, turnover was defined as the number of GPs who leave a practice divided by the average of the number of GPs at the start and the number of GPs at the end of the year. This rate definition is similar to that used in previous studies on GPs turnover ${ }^{10}$ and the current definition used by the NHS. ${ }^{19}$

$$
\text { Turnover rate }=\frac{\text { N GPs leaving a practice in a year }}{\text { Average number of GPs in a year }} \times 100
$$

\section{Where}

Average number of GPs in a year $=($ GPs in a practice at the start of the year + GPs in a practice at the end of the year) $/ 2$;

GPs in a practice at the end of the year=Number of GPs at start of the year + Number of joiners-Number of leavers.

Furthermore, with the aim of having a comprehensive picture on the movement of GPs, two additional measures were calculated: joiners' and retention rates, which describe (i) the proportion of GPs who join a practice during the year and (ii) the proportion of GPs who stay in a practice for the entire year, respectively. Therefore, while retention indicates the ability of a practice to retain its staff, a high rate of joiners is likely to generate a high rate of turnover due to the association of low tenure with likelihood to quit. Rates and statistical analyses of turnover are presented in the 
main paper, whereas retention and joiners' rates are described and reported in online supplemental tables 1 and 2.

\section{Data sources}

\section{GP workforce dataset}

General practices are required to provide data about staff working at NHS practices or other primary care organisations in England. NHS Digital, previously the Health and Social Care Information Centre, regularly publishes workforce datasets which include information on individual GPs and practice-level characteristics since 1995. These datasets are publicly available on the NHS Digital website. ${ }^{20}$ This study used the annual datasets (September releases) between 2007 and 2020 and the files containing practice-level data containing all the information relative to a practice.

\section{Membership of practices}

GPs in England are issued a code when they start prescribing, the GPs Primary General National Code (GNC), which is associated with their main prescribing cost centre and is issued by the NHS Prescription Services (NHS RxS). These codes are published by the NHS ODS on behalf of the NHS RxS. In particular, information about individual prescribing code of GPs (GNC) and the date a GP has joined and left a practice, are included in the General Medical Practitioners data and the General Medical Practices, GPs-by-general practice data (GP membership-epracmem), respectively. In these data, each GP has an entry for every main prescribing cost centre (GP practice) where they have worked. Dates of when a GP joins and leaves a GP practice enables the calculation of GP turnover across a specified time window. These datasets include information only on those GPs who can prescribe, that is, GP partners and salaried GPs. These data are published free of charge and capture information on GP membership to each practice from 1974 and are updated weekly on the NHS Technology Reference data Update Distribution ${ }^{21}$ website. Data on GP membership of practices were extracted on the second week of November 2020. Online supplemental figure 1 summarises the data process.

\section{Study design and study population}

Practice-level GP workforce data were linked to the GPsby-GPs practice data (GP membership-epracmem) using the practice code and each year included practices that were common in both data sources. The practice-level GP workforce files were used to identify practice characteristics and the GPs-by-GPs practice data (GP membershipepracmem) to calculate turnover rates combined for GP partners and salaried GPs given that these are the only GPs able to prescribe and whose information is included in the datasets. Joiners' and retention rates are described and reported in online supplemental tables 1 and 2, respectively.

\section{Statistical analyses}

Only practices with at least 750 registered patients were included in the analyses. Smaller practices were omitted $(714,8.1 \%$, practices during the entire study window) as they could have been reducing patient numbers in preparation for closure which itself would affect GP turnover; or they could be newly formed practices which might have exhibited different recruiting behaviours. Finally, practices with no GPs left at the end of the year in question (because they were closing in the following years) were excluded from the analyses (2006, 22.8\% practices during the entire time window). A table with the distribution of these practices and their turnover rates are provided in online supplemental table 3 .

\section{GP turnover rate over time and by NHS regions}

Using the GPs-by-GPs practice data (GP membershipepracmem), turnover rates were calculated for each practice and for every year in the study window (20072019). To summarise GP movement, the following analyses were performed. First, summary statistics including mean (SD), 25th, 50th, 75th percentiles were calculated and violin plots produced. Violin plots are similar to box plots (including the median as a marker and a box indicating the IQR), but overlaid with the distribution of the data for better visualisation. Second, the proportion of practices with low, medium, high and very high turnover rate (equal to $0 \%$, between $0 \%$ and $<10 \%$, between $10 \%$ and $<40 \%$ and $\geq 40 \%$, respectively) were computed for every year. Although arbitrary, these thresholds were chosen to understand better the extent of turnover and whether there was a high proportion of practices with extreme values. Third, the proportion of practices with persistent high turnover $(>10 \%)$ across 2 , 3,4 and 5 years window was calculated with the intent to explore whether practice turnover might have indicated a temporary situation (2 or 3 years persistent high turnover) or a continuing problem ( 4 or 5 years persistent high turnover). Finally, turnover rates were produced at regional level using the most recent classification of NHS region and rates compared between 2007 and 2019.

\section{Predictors of GP turnover rates}

To identify factors influencing turnover, count data models were fitted to estimate incidence rate ratios (IRR) and 95\% CIs of turnover rates. Specifically, a negative binomial distribution model was employed, this is the most appropriate model in the presence of overdispersion of the data. To explore the hypothesis that social deprivation of people living in the area where a practice was located is likely to increase turnover, the variables included in the primary model were average levels of deprivation where the practice was located (Index of Multiple Deprivation, IMD, 2015) categorised in quintiles and year in the study window. There was a small proportion of missing data 
for IMD $(0.08 \%)$ in the main analysis and type of contract $(0.30 \%)$ in the sensitivity analyses. For these variables, an extra category was included to indicate a missing value.

Multiple sensitivity analyses were performed to test the robustness of the results. (i) Restricting the analysis to practices active for the entire time-window to check whether opening or closing practices affected turnover rates. (ii) Excluding from the analysis practices that had an Alternative Provider Medical Service (APMS) contract rather than those having a General Medical Service or Personal Medical Service contract. This allowed us to explore whether turnover rates were affected by the type of contract of a practice. (iii) Fitting a random effect model with NHS region as random effect to understand whether regional variability influenced turnover rates. (iv) Restricting the analysis to 2015-2019 given that more information was available for this time-window and it was possible to include additional variables in the model other than practice-area social deprivation (IMD 2015) and year. These variables were full-time-equivalent (FTE) per 1000 patients ratio and proportion of salaried GPs in the practice, which were included to explore whether GPs workload and practice network structure (with salaried GPs more likely to leave) were associated with levels of turnover.

\section{Patients and public involvement}

Patients and public involvement (PPI) members were involved in the project. They did not contribute to the research question or study design, but provided feedback on the study findings. In particular, a forum group was organised with five PPI members. They agreed that GPs leaving a practice had a negative influence on patients' quality and continuity of care. They highlighted the following points regarding the potential disruption of their relationship with their GP: lack of communication and feeling apprehensive when they had to meet a new or different GP. Overall a personal relationship with the GP was very important, although often practices did not meet patients' expectations.

\section{RESULTS}

\section{GP turnover rates and their trends over time and by NHS region}

After merging the GP workforce data with the GPsby-GPs practice data (GP membership-epracmem), the number of practices included in the analyses decreased during the study window, from 8085 practices in 2007 to 6598 in 2019 (table 1). Online supplemental figure 1 summarises the data process.

Overall, half of the practices had zero turnover rate within each year of analysis. Over time, turnover rates increased during the study window; in particular, in 2009 , the 75 th percentile corresponded to an $11 \%$ rate and this had increased to $14 \%$ in 2019 . However, the increase was not linear as the peak occurred in 2013-2014 when the 75th percentile of turnover corresponded to $18 \%$. Summary statistics for turnover rates over time are reported in table 1 and by violin plots in online supplemental figure 2.

Between 2007 and 2019 the proportion of practices with low turnover (equal to $0 \%$, meaning that no GP left the practice that year) decreased from $79 \%$ (in 2007 ) to $61 \%$ (in 2019), whereas the proportion of practices with medium turnover rates (below 10\%) slightly increased from $1 \%$ in 2007 to $5 \%$ in 2009. Overall, $14 \%$ of the practices had high turnover (corresponding to $10 \%-40 \%$ ) in 2007 a share that increased to $27 \%$ in 2019. Approximately $8 \%$ of the practices showed very high turnover (above 40\%) during the entire time window (figure 1, table 2).

\begin{tabular}{lccccccc}
\hline Table 1 & General practitioner turnover rates between 2007 and 2019 & & & \\
\hline Year & N practices & Mean & SD & Median & IQR & 25th percentile & 75th percentile \\
\hline 2007 & 8085 & 6.9 & 18.0 & 0.0 & 0.0 & 0.0 & 0.0 \\
2008 & 8053 & 7.5 & 18.2 & 0.0 & 0.0 & 0.0 & 0.0 \\
2009 & 8077 & 9.2 & 20.3 & 0.0 & 11.1 & 0.0 & 11.1 \\
2010 & 8058 & 9.4 & 20.0 & 0.0 & 11.8 & 0.0 & 11.8 \\
2011 & 8009 & 10.5 & 20.8 & 0.0 & 15.4 & 0.0 & 15.4 \\
2012 & 7924 & 10.8 & 20.2 & 0.0 & 16.7 & 0.0 & 16.7 \\
2013 & 7809 & 11.6 & 19.3 & 0.0 & 18.2 & 0.0 & 18.2 \\
2014 & 7629 & 12.0 & 19.9 & 0.0 & 18.2 & 0.0 & 18.2 \\
2015 & 7404 & 9.4 & 17.4 & 0.0 & 14.5 & 0.0 & 14.5 \\
2016 & 7211 & 9.4 & 18.0 & 0.0 & 14.3 & 0.0 & 14.3 \\
2017 & 6963 & 9.6 & 17.2 & 0.0 & 15.4 & 0.0 & 15.4 \\
2018 & 6757 & 10.3 & 17.0 & 0.0 & 16.7 & 0.0 & 16.7 \\
2019 & 6598 & 9.1 & 16.0 & 0.0 & 14.3 & 0.0 & 14.3 \\
\hline
\end{tabular}




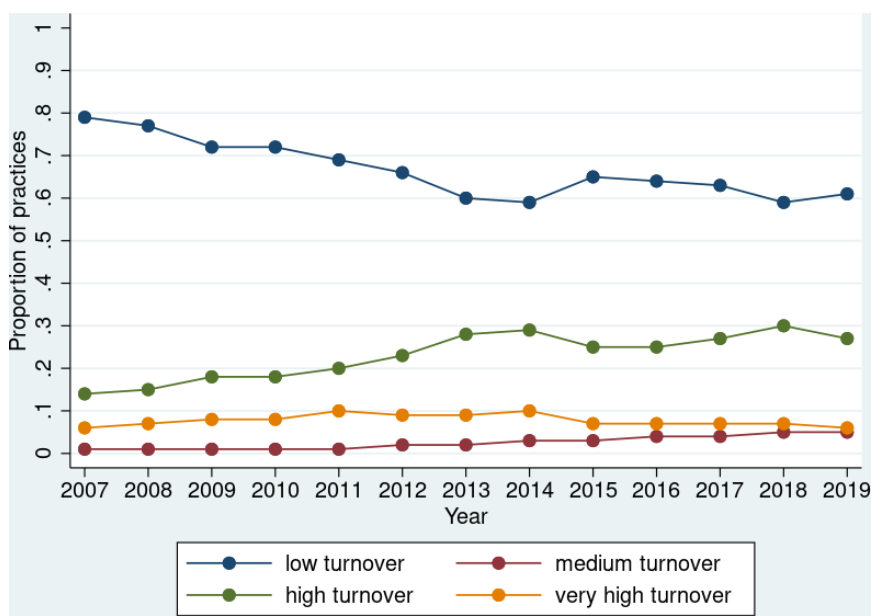

Figure 1 Proportion of practices with low, medium, high and very high turnover over time. The proportion of practices on y-axis has not been multiplied by 100 .

Turnover rates showed great variation across regions. When regional turnover rates were compared at the beginning and end of the study window, all the NHS regions had an increase in turnover rates (2007 vs 2019) (figure 2). NHS England Midlands and East (West Midlands) had the largest increase in turnover rate (on average 6\%), from $6 \%$ in 2007 to $12 \%$ in 2019; whereas NHS England Lancashire and South Cumbria had nearly no increase in turnover (on average $0 \%$ ) (figure 2). For all the regions, trends of turnover were not always consistently increasing but demonstrated peaks around 2013-2014.

Finally, when examining persistent high turnover over time $(>10 \%)$, findings revealed that, between 2007 and 2013, there had been a steady increase in the

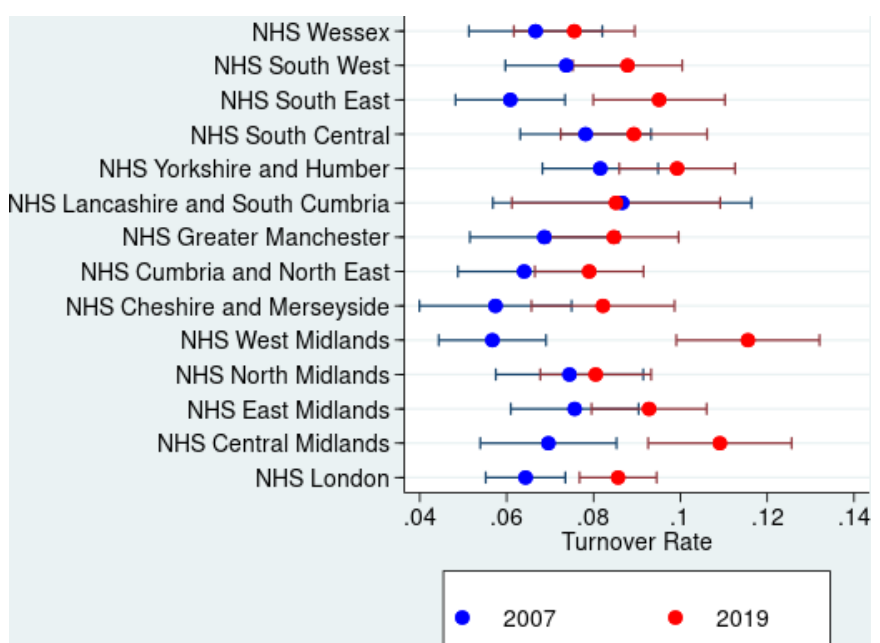

Figure 2 Comparison of general practitioners turnover rate according to National Health Service (NHS) region 2007 versus 2019. The proportion of practices on $y$-axis has not been multiplied by 100 .

proportion of practices with persistent high turnover either for 2-3 consecutive years (temporary situation) or for 4-5 consecutive years (continuing problem). Practices with high turnover over 2 years, for example, increased from $6.0 \%$ in 2007 to $17.6 \%$ in 2013, before decreasing to $14.4 \%$ in 2018 (figure 3, online supplemental table 4).

\section{Predictors of GPs turnover}

The statistical analyses investigating predictors of GPs turnover revealed that area-deprivation of a practice and year were associated with turnover rate. In particular, practices in the most deprived locations had a greater risk of higher turnover compared with

Table 2 Proportion of practices with low, medium, high and very high turnover rates over time

\begin{tabular}{|c|c|c|c|c|c|c|c|c|c|}
\hline Year & N practices & $\begin{array}{l}\mathrm{N} \text { practices } \\
\text { with low } \\
\text { turnover }\end{array}$ & $\begin{array}{l}\text { Practices } \\
\text { with low } \\
\text { turnover } \\
(\%)\end{array}$ & $\begin{array}{l}\mathrm{N} \text { practices } \\
\text { with } \\
\text { medium } \\
\text { turnover }\end{array}$ & $\begin{array}{l}\text { Practices } \\
\text { with } \\
\text { medium } \\
\text { turnover } \\
(\%)\end{array}$ & $\begin{array}{l}\mathrm{N} \text { practices } \\
\text { with high } \\
\text { turnover }\end{array}$ & $\begin{array}{l}\text { Practices } \\
\text { with high } \\
\text { turnover } \\
(\%)\end{array}$ & $\begin{array}{l}N \text { practices } \\
\text { with very } \\
\text { high } \\
\text { turnover }\end{array}$ & $\begin{array}{l}\text { Practices } \\
\text { with very } \\
\text { high } \\
\text { turnover } \\
(\%)\end{array}$ \\
\hline 2007 & 8075 & 6419 & 79.5 & 71 & 0.9 & 1104 & 13.7 & 481 & 5.96 \\
\hline 2008 & 8053 & 6208 & 77.1 & 95 & 1.2 & 1201 & 14.9 & 549 & 6.82 \\
\hline 2009 & 8077 & 5850 & 72.4 & 117 & 1.4 & 1448 & 17.9 & 662 & 8.20 \\
\hline 2010 & 8056 & 5817 & 72.2 & 107 & 1.3 & 1453 & 18.0 & 679 & 8.43 \\
\hline 2011 & 8008 & 5538 & 69.2 & 90 & 1.1 & 1588 & 19.8 & 792 & 9.89 \\
\hline 2012 & 7924 & 5218 & 65.9 & 127 & 1.6 & 1858 & 23.4 & 721 & 9.10 \\
\hline 2013 & 7808 & 4713 & 60.4 & 192 & 2.5 & 2171 & 27.8 & 732 & 9.38 \\
\hline 2014 & 7629 & 4482 & 58.7 & 199 & 2.6 & 2200 & 28.8 & 748 & 9.80 \\
\hline 2015 & 7404 & 4809 & 65.0 & 217 & 2.9 & 1863 & 25.2 & 515 & 6.96 \\
\hline 2016 & 7211 & 4647 & 64.4 & 256 & 3.6 & 1811 & 25.1 & 497 & 6.89 \\
\hline 2017 & 6963 & 4359 & 62.6 & 257 & 3.7 & 1884 & 27.1 & 463 & 6.65 \\
\hline 2018 & 6757 & 3975 & 58.8 & 310 & 4.6 & 2016 & 29.8 & 456 & 6.75 \\
\hline 2019 & 6597 & 4049 & 61.4 & 355 & 5.4 & 1808 & 27.4 & 385 & 5.84 \\
\hline
\end{tabular}




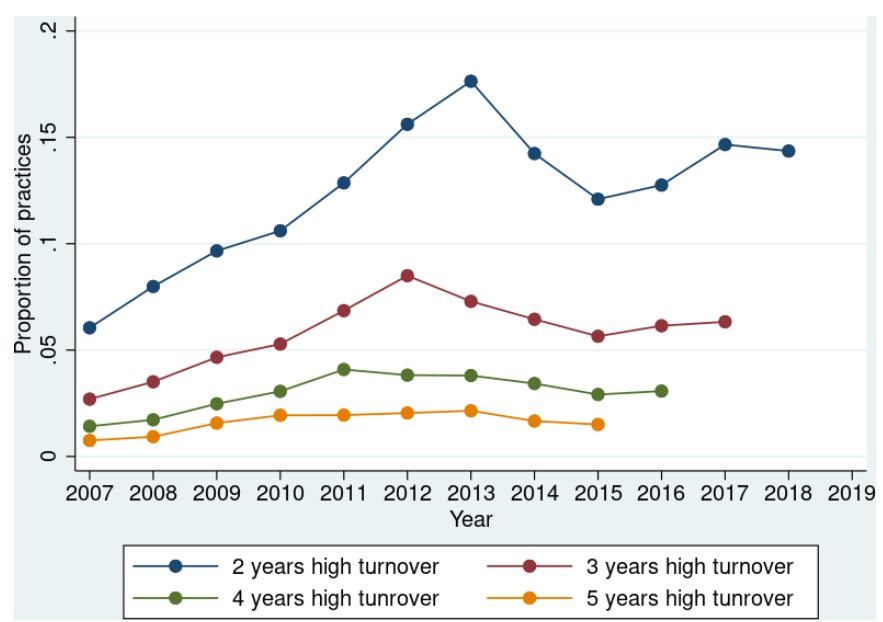

Figure 3 Proportion of practices with persistent high turnover rates $(\geq 10 \%)$ over $2,3,4$ and 5 -year window. The proportion of practices on $y$-axis has not been multiplied by 100.

practices in the least deprived areas (IRR 1.09; $95 \%$ CI 1.06 to 1.13 ); and every year in the study window was associated with an increasing turnover rate compared with 2007 (table 3), with 2013 and 2014 associated with the highest turnover rates compared with 2007 (IRR 1.67; 95\% CI 1.59 to 1.77 and IRR 1.74; $95 \%$ CI 1.64 to 1.83 ) (table 3 and figure 4 ).

Table 3 Predictors of general practitioner turnover rates (primary analysis)

\begin{tabular}{lll}
\hline & IRR $(95 \%$ Cl) & P value \\
\hline IMD & & \\
\hline 1 (least deprived) & Reference & \\
\hline 2 & $1.01(0.98$ to 1.05$)$ & 0.368 \\
\hline 3 & $1.08(1.04$ to 1.11$)$ & 0.000 \\
\hline 4 & $1.09(1.06$ to 1.13$)$ & 0.000 \\
\hline 5 (most deprived) & $1.12(1.08$ to 1.16$)$ & 0.000 \\
\hline 2007 & Reference & \\
\hline 2008 & $1.08(1.02$ to 1.15$)$ & 0.011 \\
\hline 2009 & $1.33(1.26$ to 1.41$)$ & 0.000 \\
\hline 2010 & $1.37(1.29$ to 1.45$)$ & 0.000 \\
\hline 2011 & $1.54(1.46$ to 1.63$)$ & 0.000 \\
\hline 2012 & $1.56(1.48$ to 1.65$)$ & 0.000 \\
\hline 2013 & $1.67(1.59$ to 1.77$)$ & 0.000 \\
\hline 2014 & $1.74(1.64$ to 1.83$)$ & 0.000 \\
\hline 2015 & $1.33(1.26$ to 1.41$)$ & 0.000 \\
\hline 2016 & $1.34(1.26$ to 1.41$)$ & 0.000 \\
\hline 2017 & $1.39(1.31$ to 1.47$)$ & 0.000 \\
\hline 2018 & $1.49(1.41$ to 1.58$)$ & 0.000 \\
\hline 2019 & $1.31(1.24$ to 1.39$)$ & 0.000 \\
\hline
\end{tabular}

IMD, Index of Multiple Deprivation; IRR, incidence rate ratios.

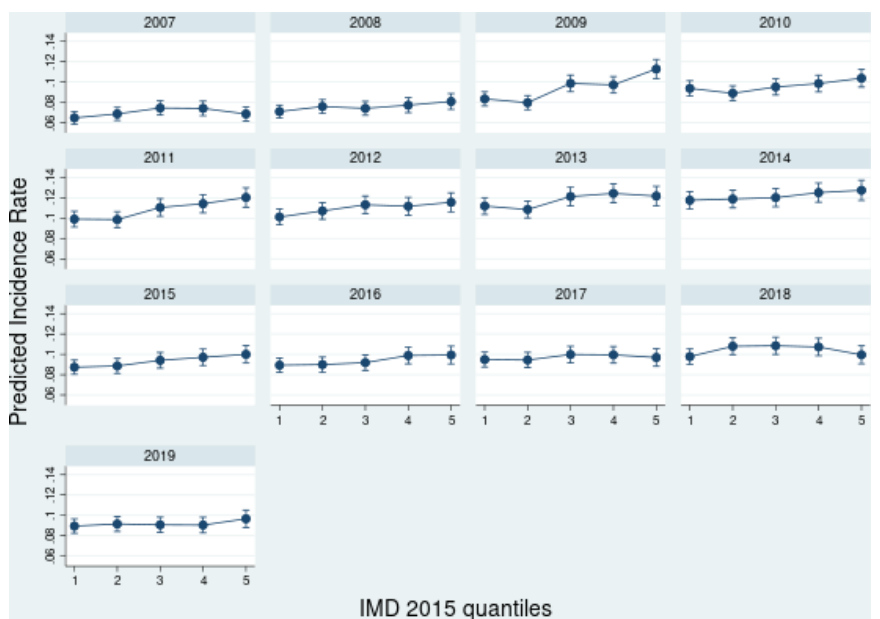

Figure 4 Predicted probabilities of general practitioner turnover according to quantiles of social deprivation (Index of Multiple Deprivation (IMD), 2015) and year. The proportion of practices on y-axis has not been multiplied by 100 .

\section{Sensitivity analyses}

Results from sensitivity analyses confirmed the main findings. This was the case when the analysis was restricted to practices active for the entire time window; or when practices with APMS contract were excluded; or when a random effect model for NHS region was fitted (table 4). When the time-window included only practices between 2015 and 2019 and additional variables were included in the statistical model, area-practice deprivation (IRR 1.08; 95\% CI 1.02 to 1.13, practices located in most deprived areas vs least deprived areas), proportion of salaried GPs (IRR 1.69; 95\% CI 1.59 to 1.79), and year 2018 compared with 2015 (IRR 1.12; 95\% CI 1.07 to 1.18) were all significantly associated with higher turnover; whereas lower workload, as expressed by the FTE per 1000 patients ratio, was associated with lower turnover rate (IRR 0.84; $95 \%$ CI 0.78 to 0.90 ) (table 5 ).

\section{DISCUSSION \\ Main findings}

For the first time, rather than intention to leave, this study describes levels of GPs turnover over a 12-year window (2007-2019) and its variation by geographical regions in English primary care. In addition, it also reports on practice-area social deprivation and practice staffing relative to patient lists that were associated with higher or lower levels of GPs turnover, respectively.

In the backdrop of a trend towards fewer and larger general practices, ${ }^{22}$ our findings revealed that turnover rates increased over the study period, although overall changes were small. Interestingly, turnover rates were the highest during 2013-2014. Over time, the proportion of practices with high turnover increased by $13 \%$ and those with very high turnover remained at the same level (around 8\%). The majority of NHS regions experienced a rise in turnover between 2007 and 2019, which was greater in some regions than others. For instance, NHS 
Table 4 Sensitivity analyses

\begin{tabular}{|c|c|c|c|c|c|c|}
\hline & \multicolumn{2}{|c|}{$\begin{array}{l}\text { Practice active for the entire time- } \\
\text { window }\end{array}$} & \multicolumn{2}{|c|}{$\begin{array}{l}\text { Excluding practices with APMS } \\
\text { contract }\end{array}$} & \multicolumn{2}{|c|}{$\begin{array}{l}\text { Random-effect model with NHS } \\
\text { region as random effect }\end{array}$} \\
\hline & IRR (95\% Cl) & $P$ value & IRR (95\% Cl) & $P$ value & IRR (95\% Cl) & P value \\
\hline \multicolumn{7}{|l|}{ IMD } \\
\hline $\begin{array}{l}1 \text { (least } \\
\text { deprived) }\end{array}$ & Reference & & & & & \\
\hline 2 & 1.01 (0.98 to 1.05$)$ & 0.386 & 1.02 (0.99 to 1.05$)$ & 0.254 & 1.02 (0.95 to 1.09$)$ & 0.583 \\
\hline 3 & 1.07 (1.04 to 1.11$)$ & 0.000 & 1.07 (1.04 to 1.11$)$ & 0.000 & 1.07 (1.01 to 1.15$)$ & 0.031 \\
\hline 4 & 1.08 (1.05 to 1.12$)$ & 0.000 & 1.09 (1.06 to 1.13$)$ & 0.000 & 1.09 (1.02 to 1.17$)$ & 0.007 \\
\hline $\begin{array}{l}5 \text { (most } \\
\text { deprived) }\end{array}$ & 1.09 (1.06 to 1.13$)$ & 0.000 & 1.11 (1.08 to 1.15$)$ & 0.000 & 1.10 (1.03 to 1.18$)$ & 0.004 \\
\hline 2007 & Reference & & & & & \\
\hline 2008 & 1.06 (0.99 to 1.12$)$ & 0.080 & 1.08 (1.01 to 1.14$)$ & 0.015 & 1.09 (0.97 to 1.22$)$ & 0.140 \\
\hline 2009 & 1.31 (1.23 to 1.39$)$ & 0.000 & 1.33 (1.26 to 1.41$)$ & 0.000 & 1.34 (1.20 to 1.50$)$ & 0.000 \\
\hline 2010 & 1.35 (1.27 to 1.43 ) & 0.000 & 1.35 (1.28 to 1.43$)$ & 0.000 & 1.36 (1.22 to 1.52$)$ & 0.000 \\
\hline 2011 & 1.53 (1.44 to 1.62$)$ & 0.000 & 1.52 (1.44 to 1.61$)$ & 0.000 & 1.53 (1.37 to 1.70$)$ & 0.000 \\
\hline 2012 & 1.56 (1.47 to 1.65$)$ & 0.000 & 1.55 (1.46 to 1.64$)$ & 0.000 & 1.57 (1.41 to 1.74$)$ & 0.000 \\
\hline 2013 & 1.65 (1.56 to 1.75$)$ & 0.000 & 1.65 (1.57 to 1.75$)$ & 0.000 & 1.68 (1.51 to 1.86$)$ & 0.000 \\
\hline 2014 & 1.70 (1.61 to 1.80$)$ & 0.000 & 1.73 (1.63 to 1.82$)$ & 0.000 & 1.74 (1.56 to 1.93 ) & 0.000 \\
\hline 2015 & 1.31 (1.23 to 1.39$)$ & 0.000 & 1.31 (1.24 to 1.39$)$ & 0.000 & 1.37 (1.22 to 1.53$)$ & 0.000 \\
\hline 2016 & 1.31 (1.24 to 1.39$)$ & 0.000 & 1.31 (1.24 to 1.39$)$ & 0.000 & 1.37 (1.22 to 1.53$)$ & 0.000 \\
\hline 2017 & 1.40 (1.32 to 1.48$)$ & 0.000 & 1.39 (1.31 to 1.47$)$ & 0.000 & 1.40 (1.25 to 1.57$)$ & 0.000 \\
\hline 2018 & 1.53 (1.44 to 1.61$)$ & 0.000 & 1.49 (1.41 to 1.57$)$ & 0.000 & 1.49 (1.34 to 1.67$)$ & 0.000 \\
\hline 2019 & $1.33(1.26$ to 1.41$)$ & 0.000 & 1.31 (1.23 to 1.38$)$ & 0.000 & 1.33 (1.18 to 1.49$)$ & 0.000 \\
\hline
\end{tabular}

Predictors of general practitioner turnover rates: (i) restricting the analysis to practices active for the entire time-window; (ii) excluding practices with APMS contract; (iii) random-effect model with NHS region as random effect.

APMS, Alternative Provider Medical Service; IMD, Index of Multiple Deprivation; IRR, incidence rate ratios; NHS, National Health Service.

Table 5 Sensitivity analyses

\begin{tabular}{|lll}
\hline & IRR (95\% CI) & P value \\
\hline IMD & & \\
\hline 1 (least deprived) & Reference & \\
\hline 2 & $1.05(1.00$ to 1.10$)$ & 0.063 \\
\hline 3 & $1.06(1.01$ to 1.12$)$ & 0.012 \\
\hline 4 & $1.06(1.01$ to 1.12$)$ & 0.013 \\
\hline 5 (most deprived) & $1.08(1.02$ to 1.13$)$ & 0.004 \\
\hline Proportion of salaried GPs & $1.69(1.59$ to 1.79$)$ & 0.000 \\
\hline FTE per 1000 patients ratio & $0.84(0.78$ to 0.90$)$ & 0.000 \\
\hline 2015 & Reference & \\
\hline 2016 & $0.99(0.95$ to 1.05$)$ & 0.845 \\
\hline 2017 & $1.03(0.98$ to 1.09$)$ & 0.215 \\
\hline 2018 & $1.12(1.07$ to 1.18$)$ & 0.000 \\
\hline 2019 & $0.98(0.93$ to 1.03$)$ & 0.504 \\
\hline
\end{tabular}

Predictors of GPs turnover restricting the time-window to 20152019 and adding 'proportion of salaried GPs' and 'FTE per 1000 patients ratio' to the model.

FTE, full-time-equivalent; GP, general practitioner; IMD, Index of Multiple Deprivation; IRR, incidence rate ratios.
England West Midlands was the region worst affected. Results also showed that, over time, there was a rising number of practices with persistent high turnover for at least five consecutive years, indicative of a continuing problem for these practices. However, this was not associated with practice-level deprivation (results not shown). Finally, there was a significant association between practice area social deprivation and levels of turnover rates. Specifically, practices located in the most deprived areas were associated with the likelihood of higher GP turnover compared with practices located in the least deprived areas.

\section{Strengths and limitations of the study}

The study has several strengths. First, it quantified and described GPs' actual turnover rather than 'intentions to leave' usually reported in existing studies. ${ }^{2}{ }^{23}$ Second, it used two national administrative datasets, regularly updated and monitored by NHS Digital and the RxS/ NHS ODS, which have the advantage of including everyone rather than only respondents to a survey, therefore they might be less prone to bias. Third, the study provided rates for a 12-year window and across regions of England. Fourth, the approach employed to calculate 
turnover rates, used the exact dates when a GP joined and left a practice, therefore more accurate than using aggregate data. ${ }^{10}$ Fifth, it presented methodological advances in combining multiple data sources containing information about the primary care workforce and historical data of individual GPs' characteristics.

Limitations of the study need to be acknowledged as well. Despite the fact the workforce data provide a wealth of information on practices characteristics, the main analyses performed in the study included only basic variables (such as practice area social deprivation and year). This was due to NHS Digital employing a revised methodology to calculate some variables (such as GPs' FTE) from 2015, therefore these data are not comparable with previous years. In addition, it was not possible to have detailed information on individual GP demographics (age and gender) and their employment model from the GP workforce datasets, therefore all the rates presented are combined for GP partners and salaried GPs. CIs for the proportions are not reported since the sample is large and there is very little uncertainty around the estimates. Hence, these would add complexity but little to no new information. Finally, it was not possible to distinguish between those GPs who moved to a different practice, retired or left primary care completely. Nevertheless, joiners' and retention rates have also been provided (online supplemental material) to give a comprehensive description of the GPs workforce behaviour of joining, staying or leaving a practice.

\section{Comparison with other studies}

Studies examining GP turnover rates in England are scarce and relate to the early 90 s. $^{1024}$ Compared with Taylor and Leese, ${ }^{10}$ turnover rates are slightly higher in our study, but this can be attributed to the different time-window analysed or to differences in the methodology used, such as combining multiple data sources, using the exact date a GP has joined or left a practice and including all types of working patterns rather than those GPs practicing full time only. ${ }^{10}$ Similar to their findings is the variation of turnover by region and its association with social deprivation. ${ }^{10}$ Increasing turnover and regional variation have also been found across NHS Trusts in England for other healthcare professionals. ${ }^{25}$ Our findings also need to be evaluated in the context of rates of intentions to leave direct patient care within 5 years, as reported in national GP surveys. ${ }^{2}$ We cannot directly compare the rates we report and those from the surveys, since we cannot quantify those who leave direct patient care, only practice turnover, and we measure that annually, not over 5 years. However, there was discrepancy in trends, with 'intention to leave' rates increasing from $19.4 \%$ in 2005 to $39 \%$ in 2017 , and we would have expected a much larger increase in turnover if the intentions reported were fully followed through. Alternatively, perhaps there is an imminent large increase in turnover expected by 2022 .

\section{Interpretation of findings and implication for practice}

Findings from our study have revealed that there was an increase in GP turnover over the last decade. This trend may be partially explained by the rising number of GPs intending to leave their profession or having a career break, ${ }^{23}$ although there was a discrepancy in rates as previously described. Burnout is considered a key factor contributing to this intention, ${ }^{26}$ known to be driven by increasing workload through patients with complex needs,${ }^{27}$ although the link to turnover is tenuous. ${ }^{28}$ Other factors relevant to turnover include: lack of or reduced job satisfaction, ${ }^{82}$ dissatisfaction with the 'amount of responsibility given,', "physical working conditions, ${ }^{2}$ and time spent on 'unimportant tasks'. ${ }^{8}$ The reasons behind the peak of turnover in 2013-2014 are unclear; this coincides with the introduction of the APMS contract, but we could not confirm causality.

Existing literature highlights that GPs often find managing patients in areas of socioeconomic deprivation a challenge due to the higher prevalence of multimorbidity and the associated healthcare needs. ${ }^{29-31}$ The higher turnover rates observed in more deprived areas might also be related to differences in the distribution of GP or other healthcare professional workforce, though it is difficult to determine whether these differences are the cause or consequence of higher GP turnover.

Regional variations in turnover might be due to different levels of social deprivation across the regions and varying health services' pressures. Whereas, the persistent high turnover experience by a number of practices, indicative of a continuing and unresolved problem within the practice or area rather than temporary situation, might be associated with practices experiencing problems with recruitment and retention for specific reasons. ${ }^{10}$ There is also variation in the characteristics of the GPs across regions, with some regions being served by older or overseas qualified GPs, who may be more mobile. ${ }^{32}$

High or increasing GP turnover is a concern for the entire healthcare system, especially considering existing difficulties in replacing retiring GPs. ${ }^{32}$ Recently, the ReGROUP project concluded that policies and strategies to address the existing healthcare workforce crisis in primary care and maximise retention of GPs should facilitate sustainable GP workload and contractual requirements, as well as the need for personal and professional support; in addition to target areas which influence job satisfaction and work-life balance. ${ }^{33}$

\section{CONCLUSIONS}

We observed a small overall increase in GP turnover in the last decade across the whole of England, supporting previous local investigations and national surveysalthough that increase was not linear, with a turnover peak in 2013-2014, coinciding with the introduction of the APMS contract. Greater attention to GP turnover is needed, particularly in the most severely deprived areas, to address the complex health needs of the population 
living in these areas and avoid the exacerbation of health inequalities. Moreover, there is a large cost associated with GP turnover and practices with very high persistent turnover need to be further investigated. Finally, targeted policies and strategies need to be developed and tested to diminish its occurrence.

\section{Author affiliations}

${ }^{1}$ Division of Informatics, Imaging \& Data Sciences, School of Health Sciences, The University of Manchester, Manchester, UK

${ }^{2}$ NIHR School for Primary Care Research, Division of Population Health, Health Services Research and Primary Care, The University of Manchester, Manchester, UK ${ }^{3}$ Health Organisation, Policy and Economics (HOPE) Group, Centre for Primary Care \& Health Services Research, The University of Manchester, Manchester, UK ${ }^{4}$ Alliance Manchester Business School, The University of Manchester, Manchester, UK

${ }^{5} \mathrm{NIHR}$ Greater Manchester Patient Safety Translational Research Centre, The University of Manchester, Manchester, UK

\section{Twitter Peter Bower @Bowercpcman and Evangelos Kontopantelis @dataevan}

Contributors PB, KC, JR, MS, AE, SS, Y-SL, SJG and EK secured funding for the study. RP, YSL and EK designed the analyses. RP performed the statistical analyses with input from PB, KC, JR, MS, AE, SS, Y-SL, SJG and EK. RP drafted the paper and $\mathrm{PB}, \mathrm{KC}, \mathrm{JR}, \mathrm{MS}, \mathrm{AE}, \mathrm{SS}, \mathrm{Y}-\mathrm{SL}, \mathrm{SJG}$ and EK critically revised it. RP is the guarantor. The corresponding author attests that all listed authors meet authorship criteria and no others meeting the criteria have been omitted.

Funding This project has been funded by the Health Foundation as part of the Efficiency Research Programme (AIMS ID: 1318316). All decisions concerning analysis, interpretation and publication are made independently from the funder.

Competing interests None declared.

Patient consent for publication Not required.

Provenance and peer review Not commissioned; externally peer reviewed.

Data availability statement Data are available in a public, open access repository. The GP workforce and the GPs-by-general practices data are freely available from the NHS Digital and TRUD websites, respectively.

Supplemental material This content has been supplied by the author(s). It has not been vetted by BMJ Publishing Group Limited (BMJ) and may not have been peer-reviewed. Any opinions or recommendations discussed are solely those of the author(s) and are not endorsed by BMJ. BMJ disclaims all liability and responsibility arising from any reliance placed on the content. Where the content includes any translated material, BMJ does not warrant the accuracy and reliability of the translations (including but not limited to local regulations, clinical guidelines, terminology, drug names and drug dosages), and is not responsible for any error and/or omissions arising from translation and adaptation or otherwise.

Open access This is an open access article distributed in accordance with the Creative Commons Attribution Non Commercial (CC BY-NC 4.0) license, which permits others to distribute, remix, adapt, build upon this work non-commercially, and license their derivative works on different terms, provided the original work is properly cited, appropriate credit is given, any changes made indicated, and the use is non-commercial. See: http://creativecommons.org/licenses/by-nc/4.0/.

\section{ORCID iDs}

Sharon Spooner http://orcid.org/0000-0001-6965-3673

Evangelos Kontopantelis http://orcid.org/0000-0001-6450-5815

\section{REFERENCES}

1 RCGP. New League table reveals GP shortages across England, as patients set to wait week or more to see family doctor on $67 \mathrm{M}$ occasions, 2015. Available: https://healthwatchtrafford.co.uk/news/ new-league-table-reveals-gp-shortages-across-england-as-patientsset-to-wait-week-or-more-to-see-family-doctor-on-67m-occasions/ [Accessed $22 \mathrm{Jul}$ 2020].

2 Gibson J, Sutton M, Spooner S. University of Manchester: Policy Research Unit in Commissioning and the Healthcare System Manchester Centre for Health Economics. In: Ninth national GP Worklife survey, 2018: 36.
3 Martin S, Davies E, Gershlick B. Under pressure: What the Commonwealth Fund's 2015 international survey of general practitioners means for the UK. Health Foundation, 2016.

4 Shen $\mathrm{X}$, Jiang $\mathrm{H}, \mathrm{Xu} \mathrm{H}$, et al. The global prevalence of turnover intention among general practitioners: a systematic review and metaanalysis. BMC Fam Pract 2020;21:246.

5 Hayes H, Gibson J, Fitzpatrick B, et al. Working lives of GPs in Scotland and England: cross-sectional analysis of national surveys. BMJ Open 2020;10:e042236.

6 Buchan J, Charlesworth A, Gershlick B. A critical moment: NHS staffing trends, retention and attrition, 2019.

7 Hunt J. New deal for general practice. Jeremy Hunt sets out the first steps in a new deal for GPs, 2015.

8 Dale J, Potter R, Owen K, et al. Retaining the general practitioner workforce in England: what matters to GPs? A cross-sectional study. BMC Fam Pract 2015;16:140.

9 Fletcher E, Abel GA, Anderson R, et al. Quitting patient care and career break intentions among general practitioners in South West England: findings of a census survey of general practitioners. $B M J$ Open 2017;7:e015853.

10 Taylor DH, Leese B. General practitioner turnover and migration in England 1990-94. Br J Gen Pract 1998;48:1070-2.

11 Reddy A, Pollack CE, Asch DA, et al. The effect of primary care provider turnover on patient experience of care and ambulatory quality of care. JAMA Intern Med 2015;175:1157-62.

12 Fan VS, Burman M, McDonell MB, et al. Continuity of care and other determinants of patient satisfaction with primary care. $J$ Gen Intern Med 2005;20:226-33.

13 Hussey PS, Schneider EC, Rudin RS, et al. Continuity and the costs of care for chronic disease. JAMA Intern Med 2014;174:742-8.

14 Nyweide DJ, Anthony DL, Bynum JPW, et al. Continuity of care and the risk of preventable hospitalization in older adults. JAMA Intern Med 2013:173:1879-85.

15 Buchbinder SB, Wilson M, Melick CF, et al. Primary care physician job satisfaction and turnover. Am J Manag Care 2001;7:701-13.

16 Shanafelt T, Goh J, Sinsky C. The business case for investing in physician well-being. JAMA Intern Med 2017;177:1826-32.

17 Buchan J. Reviewing the benefits of health workforce stability. Hum Resour Health 2010;8:29.

18 Hann M, Reeves D, Sibbald B. Relationships between job satisfaction, intentions to leave family practice and actually leaving among family physicians in England. Eur $J$ Public Health 2011;21:499-503.

19 NHS Digital. Joiners and leaver rates with stability index 2016 -2018. Available: https://digital.nhs.uk/data-and-information/find-dataand-publications/supplementary-information/2019-supplementaryinformation-files/leavers-and-joiners/joiners-and-leaver-rates-withstability-index-2016-2018

20 NHS Digital. General practice workforce. Available: https://digital. nhs.uk/data-and-information/publications/statistical/general-andpersonal-medical-services

21 NHS Digital TRUD. NHS Ods Weekly prescribing-related data. Available: https://isd.digital.nhs.uk/trud3/user/guest/group/0/pack/5

22 PULSE. Average practice list size grows by more than $2 \%$ in just eight months. Available: https://www.pulsetoday.co.uk/news/ practice-closures/average-practice-list-size-grows-by-more-than-2in-just-eight-months/

23 Gibson J, Checkland K, Coleman A. Eighth national GP Worklife survey, 2015.

24 Taylor DH, Leese B. Recruitment, retention, and time commitment change of general practitioners in England and Wales, 1990-4: a retrospective study. BMJ 1997;314:314.

25 Buchan J, Charlesworth A, Gershlick B. Rising pressure: the NHS workforce challenge 2017.

26 Cheshire A, Ridge D, Hughes J, et al. Influences on GP coping and resilience: a qualitative study in primary care. $\mathrm{Br} J$ Gen Pract 2017;67:e428-36.

27 Pedersen AF, Nørøxe KB, Vedsted P. Influence of patient multimorbidity on GP burnout: a survey and register-based study in Danish general practice. Br J Gen Pract 2020;70:e95-101.

28 Pedersen AF, Andersen CM, Olesen F, et al. Risk of burnout in Danish GPs and exploration of factors associated with development of burnout: a two-wave panel study. Int J Family Med 2013;2013:1-8.

29 Eley E, Jackson B, Burton C, et al. Professional resilience in GPs working in areas of socioeconomic deprivation: a qualitative study in primary care. Br J Gen Pract 2018;68:e819-25.

30 O'Brien R, Wyke S, Guthrie B, et al. An 'endless struggle': a qualitative study of general practitioners' and practice nurses' experiences of managing multimorbidity in socio-economically deprived areas of Scotland. Chronic IIIn 2011;7:45-59. 
31 Long L, Moore D, Robinson S, et al. Understanding why primary care doctors leave direct patient care: a systematic review of qualitative research. BMJ Open 2020;10:e029846.

32 Esmail A, Panagioti M, Kontopantelis E. The potential impact of Brexit and immigration policies on the GP workforce in England: a cross-sectional observational study of GP qualification region and the characteristics of the areas and population they served in September 2016. BMC Med 2017;15:191.

33 Chilvers R, Richards SH, Fletcher E, et al. Identifying policies and strategies for general practitioner retention in direct patient care in the United Kingdom: a RAND/UCLA appropriateness method panel study. BMC Fam Pract 2019;20:130. 